\title{
SUSTAINABILITY OF CITRUS RETICULATA L. BLANCO. AND MANGIFERA INDICA L. IS RELATED TO ANTIOXIDATIVE DEFENSE UNDER SPATIAL AND TEMPORAL FLUCTUATIONS OF SOIL AND IRRIGATION WATER
}

\author{
GHAFFAR, S. ${ }^{*}$ - MAHMOOD, S. - NoreEn, S. - AKREM, A. \\ Environment and Evolutionary Laboratories (EEL), Institute of Pure and Applied Biology, \\ Bahauddin Zakariya University, Multan, Pakistan \\ *Corresponding author \\ e-mail: ghaffarshazia@gmail.com \\ (Received $18^{\text {th }}$ Mar 2021; accepted $29^{\text {th }}$ Jul 2021)
}

\begin{abstract}
Spatial and temporal variability for the quality of surface and ground irrigation water and its impact on soil characteristics were assessed for local farms of Citrus reticulata L. Blanco. (Citrus) and Mangifera indica L. (Mango). Electrical Conductivity (EC), Potential Hydrogen (pH), Sodium $\left(\mathrm{Na}^{+}\right.$), Calcium magnesium $\left(\mathrm{Ca}^{+2}+\mathrm{Mg}^{+2}\right)$, Chromium $(\mathrm{Cr})$, Lead $(\mathrm{Pb})$, Nickel $(\mathrm{Ni})$, Zinc $(\mathrm{Zn})$, Biological Oxygen Demand (BOD), Chemical Oxygen Demand (COD) were determined. Ground water at an aquifer of $(30-40 \mathrm{~cm})\left(0.57\right.$ and $\left.0.88 \mathrm{mg} \mathrm{L}^{-1}\right)$, and two depths of soil profiles $\left(0.97\right.$ and $\left.2.29 \mathrm{mg} \mathrm{kg}^{-1}\right)$ had more $\mathrm{Cr}$ and $\mathrm{Pb}$, respectively. Biochemical characteristics of the species varied spatially and temporally except the chlorophyll content. Lipid peroxidation via MDA production became evident. An enhanced activity of enzymatic Superoxide dismutase (SOD), Catalase (CAT), Peroxidase (POD) and non-enzymatic components (Ascorbic acid, Carotenoids, Phenols, Sugars) was noticed but the higher resilience in mango can be attributed to non-enzymatic defense. The bioaccumulation of $\mathrm{Cr}$ and $\mathrm{Pb}$ declined markedly $(\mathrm{P} \leq 0.001)$ in species from roots to aerial tissues. Citrus pulp contained a significant $(\mathrm{P} \leq 0.001)$ amount of $\mathrm{Cr}$, and $\mathrm{Pb}$. The study suggested strict environmental monitoring for biodiversity and conservation. However, metal accumulation in the edible portion of citrus seemed to be of great concern for food safety. Keywords: water and soil pollution, spatial and temporal variability, citrus and mango, heavy metals, food security and safety
\end{abstract}

\section{Introduction}

Agriculture serves as the backbone of the economy for many countries of the world as it contributes considerably to their GDP (Leoveanu-Soare et al., 2020). The practice of irrigation has been a central feature of agriculture for over 5,000 years. Like many other countries, Pakistan is also an agricultural country where most of the population relies on arable land for their livelihood. The cultivated area covers 22.68 million hectares out of which 25 percent is rainfed (Chaudhry, 2017).

Multan $\left(30^{\circ} 11^{\prime} 44^{\prime \prime}\right.$ North, $71^{\circ} 28^{\prime} 31^{\prime \prime}$ East) is an ancient city located in Southern Punjab, Pakistan. It is situated on the main trade route at the convergence of two main rivers; Chenab and Ravi. The area holds a traditional significance for agriculture despite arid climate with an annual rainfall $180 \mathrm{~mm}$ with summer and winter temperatures $45^{\circ} \mathrm{C}$ and $4^{\circ} \mathrm{C}$, respectively. Owing to high temperature and rate of evapotranspiration during summer months (May to October), irrigation other than precipitation becomes crucial. Thus, canal (surface) tube well (ground) water is used for irrigation, the latter being ascent through mechanical pumps. Several industries have been set up in the city that include fertilizer, vegetable oil / ghee, textile and tanneries. The effluent or discharge from these industries loaded with several contaminants containing heavy metals is directly thrown into canals or water courses without any treatment. Moreover, land fill 
by industrial and domestic wastes in peri-urban and rural areas not only contaminate soil but also ground water (Nai et al., 2021).

Citrus reticulata L. Blanco. (Citrus) and Mangifera indica L. (Mango) are the prime fruits of this region thus generate major revenue for the country through export and as such contributes towards socio-economic growth of the region (Randhawa et al., 2014). The production of citrus and mango in the world ranks $10^{\text {th }}$ and $6^{\text {th }}$ with 2.3 and 1.0 million-tons yield, respectively, during 2016 (GOP, 2016). The country earned US\$ 21 million from the export of mango alone ( $\mathrm{Naz}$ et al., 2014). Main mango producing areas in Pakistan are Punjab and Sindh with 1.2 and 0.5 million tons yield, correspondingly (Rehman et al., 2015) while citrus is mostly grown in Multan, Sahiwal and Sargodha regions.

C. reticulata Blano. belongs to the family Rutaceae and is commonly known as "Kinnow" in Pakistan. The prominent aroma and taste make it popular commodity having essential dietary requirements. About $100 \mathrm{ml}$ of Kinnow juice contains 20-25 mg of Vitamin C (Noor, 2015). M. indica L. has tropical distribution (Muniraja et al., 2019) and is a member of Anacardiaceae known as "the king of fruits". Popular edible portion has special quality attributes such as nutrition, aroma, sweetness, taste and brix with therapeutic significance.

During the past two decades, rapid industrialization and urbanization resulted in significant pollution of water, air and agricultural soil. Consequently, a considerable decline in the production of these fruits has been reported (Gupta et al., 2020) due to several types of contaminants including both essential and non-essential elements. Although essential elements play a pivotal role for growth of plant species but non-essential trace elements like Chromium (Cr), Cadmium (Cd), Mercury ( $\mathrm{Hg}$ ), and Lead $(\mathrm{Pb})$ are phytotoxic. Owing to their non-biodegradable nature they can persist ubiquitously in the environment (Sabir et al., 2015). These metal elements tend to accumulate in several plant organs that poses serious threat to production and human health consequent upon their consumption (Hu et al., 2017). Agricultural soils when irrigated with waste water get adversely contaminated with such elements. Hence, plants grown on such soils can bioaccumulate a substantial amount of these metals in their different tissues. The soils, plants and the products made thereof have been shown to be highly contaminated therefore is of immense public concern as far as food safety is concerned (Dotaniya et al., 2017). The accumulation of undesirable contents in edible plants parts also deteriorate aroma, taste and sometimes completely spoil the fruits thus result in substantial economic losses.

The productivity also declines because several vital processes associated with growth and development are considerably affected by the presence of metal contaminants in environment (Mahfooz et al., 2020). These contaminants are of hazardous nature as they induce the production of Reactive Oxygen Species (ROS) leading to oxidative damages to several major macromolecules and cellular entities in plants (Ibrahim et al., 2015). The extent of such damage is assessed via quantity of Malondialdehyde (MDA) owing to an affirmative relationship between its amount and oxidative stress (Tirani and Hoghjou, 2019).

Nevertheless, some plant species possess innate capabilities to withstand several environmental constraints (Boquete et al., 2021). In order to cope with oxidative stress, some plausible mechanisms have been evolved in plants. For scavenging of ROS, enzymatic; Super oxide dismutase (SOD), Catalase (CAT) and Peroxidase (POD) (Amjad et al., 2020) and non-enzymatic antioxidants; ascorbic acid, carotenoids, and 
phenolics and soluble sugars (Medveckiene et al., 2020) serve as ubiquitous and common defense in plants.

Keeping in view the above situation, the present study was aimed to reveal relationship between spatial and temporal variation in the quality of irrigation water, soil contamination and bioaccumulation of metal content in the selected fruit species to address food security and safety. The study will serve as a model for citrus and mango growing regions of the world with similar constraints. The foremost objective of the study was to reveal possible underlying biochemical and antioxidative system in these two species as well as to determine metal contents in the edible fruit parts to ensure food safety as per standards already reported (WHO, 2012).

\section{Material and Methods}

\section{Study area}

Site 1 (Aziz Farm) and site 2 (Nasim Farm) were selected for both fruits from Multan, Punjab, Pakistan depicted in Figures 1 and 2, respectively. The choice of these sites was based on a wide range of fluctuating features such as cold winters with ground frost having temperature around $4{ }^{\circ} \mathrm{C}$, hot summers and aridity with an average rainfall up to $186 \mathrm{~mm}$ during monsoon. Global Positioning System (GPS) was used to record the location of each sampling site as given under.

Several standard procedures were carried out for the analyses of water, soil and plant material and a summary of protocols followed are appended in Table 1.

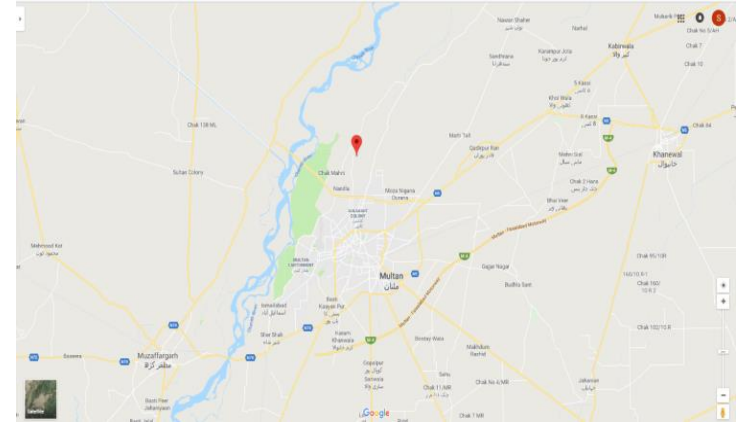

Figure 1. Location of sitel (Aziz Farm) $\left(30^{\circ} 17^{\prime} 16.6^{\prime \prime} N, 71^{\circ} 28^{\prime} 25.0^{\prime \prime} E\right)$

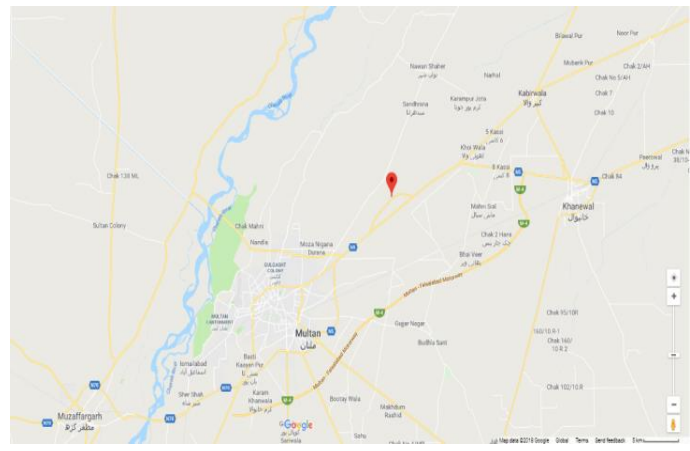

Figure 2. Location of site2 (Nasim Farm) $\left(30^{\circ} 18^{\prime} 00.9^{\prime \prime} \mathrm{N}, 71^{\circ} 39^{\prime} 01.7^{\prime \prime} \mathrm{E}\right)$

\section{Water and soil sampling}

Irrigation water samples were collected from their sources (canal and tube well) While soil samples were collected at the depths of $0-10 \mathrm{~cm}$ and $10-20 \mathrm{~cm}$ at both study sites during summer (May to October) and winter (November to March) in 2017 by using standard sampling procedures by (ICARDA Manual, Estefan et al., 2013). The water samples were collected in clean food grade plastic bottles and soil was collected in polyethylene bags, labeled appropriately then were carried back to the Environment and Evolutionary Laboratories (EEL), Institute of Pure and Applied Biology, Bahauddin Zakariya University, Multan for different physico-chemical and metal analysis. 
Table 1. Summary analysis of water, soil and plant samples with respective methodology

\begin{tabular}{c|c}
\hline Parameters & Methodology/protocols followed \\
$\mathrm{pH}$ & Richards (1954) \\
$\mathrm{EC}$ & Richards (1954) \\
$\mathrm{BOD}$ & Wei (2002) \\
$\mathrm{COD}$ & Jirka and Carter (1975) \\
$\mathrm{Na}^{+}, \mathrm{Ca}^{+2}+\mathrm{Mg}^{+2}$ & Richards (1954) \\
$\mathrm{Cr}, \mathrm{Ni}, \mathrm{Pb}, \mathrm{Zn}$ & Arora et al. (2008) \\
Chlorophyll $(a$ \& b) & Lichtenthaler (1987) \\
MDA & Cakmak and Horst (1991) \\
Enzymatic antioxidants & \\
Superoxide dismutase (SOD) & Giannopolitis and Ries (1977) \\
Catalase (CAT) & Chance and Maehly (1995) \\
Peroxidase (POD) & Chance and Maehly (1995) \\
\hline \multicolumn{2}{c}{ Non-enzymatic antioxidants } \\
\hline Ascorbic acid & Keller and Schwager (1977) \\
Total Carotenoids & Arnon (1949) \\
Total phenolic contents & Ainsworth and Gillespie (2007) \\
Soluble Sugars & Prud'homme et al. (1992)
\end{tabular}

\section{Plant tissues collection}

Roots, shoots, leaves and fruits from citrus and mango trees were taken from three fully grown trees during their respective fruiting seasons during June for mango and December, 2017 for citrus from selected sites by employing standard protocols of biochemical analysis. Three fully grown leaves were randomly chosen across the canopy of each tree starting from the base to top.

\section{Preparation of soil and plant assays}

For soil digestion, Aqua Regia method (Chen and Ma, 2001) of digestion by using hot plate was used. While the plant tissue samples were digested by employing acid digestion mixture method (Moore and Chapman, 1986).

\section{Pulp extraction and metal analysis}

The citrus and mango fruit samples from each orchard were weighed and pulp was separated by passing through a pulper. The obtained pulp was weighed and polyethylene bags were used for packing, labelled and kept in refrigerator at $4^{0} \mathrm{C}$ for 72-96 hours.

\section{Statistical analysis}

Each value in the data was across three replications that was used to calculate mean and standard error $( \pm \mathrm{S}$. E). For biochemical analyses of the plant samples, each tree was considered as an individual and three leaves were taken from each tree. Thus, a single sample is comprised of 9 leaves and each procedure was repeated three times. The data for species, sites, seasons and water sources were then subjected to a Two-Way Analysis of Variance accordingly using MS Excel 2019 and STATISTIX v8.1 in order to determine significant difference between factors separately for each parameter. Least Significant Difference (LSD) between means was calculated by applying Tukey's test (Tukey, 1949) using 5\% level of probability. 


\section{Results}

\section{Water and soil quality parameters}

Canal water samples had the highest $\mathrm{pH}$ during summer in comparison to ground water which exhibited higher value for $\mathrm{pH}$ during winter at site 1 . However, the reverse was true for site 2 with more $\mathrm{pH}$ of canal water during winter (Table 2). ANOVA for $\mathrm{pH}$ of irrigation water showed significant $(\mathrm{P} \leq 0.05)$ contrast for $\mathrm{pH}$ regarding irrigation water sources at both sites (Table 3). $\mathrm{pH}$ for soil $(0-10 \mathrm{~cm})$ depth presented in Table 4 clearly indicated high $\mathrm{pH}$ of the citrus rhizosphere during summer at both sites. The findings were comparable for $\mathrm{pH}$ at $10-20 \mathrm{~cm}$ soil depth at site 2 (Table 5). Statistical analysis (Table 3 ) revealed highly significant $(\mathrm{P} \leq 0.001)$ contrast for $\mathrm{pH}$ regarding seasons and species at both depths of both sites.

Table 2. Mean values ( \pm S.E) for physico-chemical and heavy metal contents of irrigation water samples collected for surface (canal) and ground water (tube well) during summer (May to October) and winter (November to March), at Site 1 (Aziz Farm) and Site 2 (Nasim Farm), Multan, Punjab, Pakistan

\begin{tabular}{|c|c|c|c|c|c|c|c|c|}
\hline \multirow{3}{*}{ Parameters } & \multicolumn{4}{|c|}{ Site 1} & \multicolumn{4}{|c|}{ Site 2} \\
\hline & \multicolumn{2}{|c|}{ Summer } & \multicolumn{2}{|c|}{ Winter } & \multicolumn{2}{|c|}{ Summer } & \multicolumn{2}{|c|}{ Winter } \\
\hline & $\begin{array}{l}\text { Surface } \\
\text { water }\end{array}$ & $\begin{array}{l}\text { Ground } \\
\text { water }\end{array}$ & $\begin{array}{l}\text { Surface } \\
\text { water }\end{array}$ & $\begin{array}{l}\text { Ground } \\
\text { water }\end{array}$ & $\begin{array}{l}\text { Surface } \\
\text { water }\end{array}$ & $\begin{array}{l}\text { Ground } \\
\text { water }\end{array}$ & $\begin{array}{l}\text { Surface } \\
\text { water }\end{array}$ & $\begin{array}{l}\text { Ground } \\
\text { water }\end{array}$ \\
\hline $\mathrm{pH}$ & $\begin{array}{c}8.40 \\
\pm 0.15\end{array}$ & $\begin{array}{c}6.73 \\
\pm 0.09\end{array}$ & $\begin{array}{c}8.09 \\
\pm 0.03\end{array}$ & $\begin{array}{c}7.48 \\
\pm 0.41\end{array}$ & $\begin{array}{c}8.40 \\
\pm 0.15\end{array}$ & $\begin{array}{c}6.73 \\
\pm 0.09\end{array}$ & $\begin{array}{c}8.09 \\
\pm 0.03\end{array}$ & $\begin{array}{c}7.48 \\
\pm 0.41\end{array}$ \\
\hline $\mathrm{EC}\left(\mathrm{dS} \mathrm{m} \mathrm{m}^{-1}\right)$ & $\begin{array}{c}0.46 \\
\pm 0.00\end{array}$ & $\begin{array}{c}0.70 \\
\pm 0.06\end{array}$ & $\begin{array}{c}0.45 \\
\pm 0.02\end{array}$ & $\begin{array}{c}0.66 \\
\pm 0.02\end{array}$ & $\begin{array}{c}0.46 \\
\pm 0.00\end{array}$ & $\begin{array}{c}0.70 \\
\pm 0.06\end{array}$ & $\begin{array}{c}0.45 \\
\pm 0.02\end{array}$ & $\begin{array}{c}0.66 \\
\pm 0.02\end{array}$ \\
\hline $\mathrm{BOD}\left(\mathrm{mg} \mathrm{l}^{-1}\right)$ & $\begin{array}{l}75.03 \\
\pm 0.06\end{array}$ & $\begin{array}{l}76.42 \\
\pm 0.22\end{array}$ & $\begin{array}{l}80.33 \\
\pm 0.26\end{array}$ & $\begin{array}{l}81.58 \\
\pm 0.22\end{array}$ & $\begin{array}{l}75.03 \\
\pm 0.06\end{array}$ & $\begin{array}{l}76.42 \\
\pm 0.22\end{array}$ & $\begin{array}{l}80.33 \\
\pm 0.26\end{array}$ & $\begin{array}{l}81.58 \\
\pm 0.22\end{array}$ \\
\hline $\mathrm{COD}\left(\mathrm{mg} \mathrm{l}^{-1}\right)$ & $\begin{array}{c}179.42 \\
\pm 0.34\end{array}$ & $\begin{array}{l}181.10 \\
\pm 0.24\end{array}$ & $\begin{array}{c}180.84 \\
\pm 0.48\end{array}$ & $\begin{array}{c}181.84 \\
\pm 0.24\end{array}$ & $\begin{array}{l}179.42 \\
\pm 0.34\end{array}$ & $\begin{array}{c}181.10 \\
\pm 0.24\end{array}$ & $\begin{array}{c}180.84 \\
\pm 0.48\end{array}$ & $\begin{array}{c}181.84 \\
\pm 0.24\end{array}$ \\
\hline $\mathrm{Na}^{+}\left(\mathrm{mg} \mathrm{l}^{-1}\right)$ & $\begin{array}{l}31.97 \\
\pm 1.44\end{array}$ & $\begin{array}{l}34.96 \\
\pm 7.37\end{array}$ & $\begin{array}{l}32.43 \\
\pm 2.76\end{array}$ & $\begin{array}{l}28.75 \\
\pm 3.51\end{array}$ & $\begin{array}{l}31.97 \\
\pm 1.44\end{array}$ & $\begin{array}{l}34.96 \\
\pm 7.37\end{array}$ & $\begin{array}{l}32.43 \\
\pm 2.76\end{array}$ & $\begin{array}{l}28.75 \\
\pm 3.51\end{array}$ \\
\hline $\begin{array}{c}\mathrm{Ca}^{+2}+\mathrm{Mg}^{+2} \\
\left(\text { meq } 1^{-1}\right)\end{array}$ & $\begin{array}{l}4.06 \\
\pm 0.10\end{array}$ & $\begin{array}{c}5.95 \\
\pm 0.82\end{array}$ & $\begin{array}{c}4.42 \\
\pm 0.16\end{array}$ & $\begin{array}{c}6.12 \\
\pm 0.13\end{array}$ & $\begin{array}{l}4.06 \\
\pm 0.10\end{array}$ & $\begin{array}{c}5.95 \\
\pm 0.82\end{array}$ & $\begin{array}{c}4.42 \\
\pm 0.16\end{array}$ & $\begin{array}{c}6.12 \\
\pm 0.13\end{array}$ \\
\hline $\mathrm{Cr}\left(\mathrm{mg} \mathrm{l}^{-1}\right)$ & $\begin{array}{c}0.35 \\
\pm 0.06\end{array}$ & $\begin{array}{c}0.35 \\
\pm 0.05\end{array}$ & $\begin{array}{c}0.35 \\
\pm 0.01\end{array}$ & $\begin{array}{c}0.33 \\
\pm 0.01\end{array}$ & $\begin{array}{c}0.35 \pm \\
0.06\end{array}$ & $\begin{array}{c}0.35 \\
\pm 0.05\end{array}$ & $\begin{array}{c}0.35 \\
\pm 0.01\end{array}$ & $\begin{array}{c}0.33 \\
\pm 0.01\end{array}$ \\
\hline $\mathrm{Ni}\left(\mathrm{mg} \mathrm{l}^{-1}\right)$ & $\begin{array}{c}0.22 \\
\pm 0.03\end{array}$ & $\begin{array}{c}0.45 \\
\pm 0,03\end{array}$ & $\begin{array}{c}0.21 \\
\pm 0.01\end{array}$ & $\begin{array}{c}0.44 \\
\pm 0.01\end{array}$ & $\begin{array}{c}0.22 \pm \\
0.03\end{array}$ & $\begin{array}{c}0.45 \\
\pm 0,03\end{array}$ & $\begin{array}{c}0.21 \\
\pm 0.01\end{array}$ & $\begin{array}{c}0.44 \\
\pm 0.01\end{array}$ \\
\hline $\mathrm{Pb}\left(\mathrm{mg} \mathrm{l}^{-1}\right)$ & $\begin{array}{c}0.44 \\
\pm 0.07\end{array}$ & $\begin{array}{c}0.56 \\
\pm 0.03\end{array}$ & $\begin{array}{c}0.42 \\
\pm 0,03\end{array}$ & $\begin{array}{c}0.55 \\
\pm 0.01\end{array}$ & $\begin{array}{c}0.44 \pm \\
0.07\end{array}$ & $\begin{array}{c}0.56 \\
\pm 0.03\end{array}$ & $\begin{array}{c}0.42 \\
\pm 0,03\end{array}$ & $\begin{array}{c}0.55 \\
\pm 0.01\end{array}$ \\
\hline $\mathrm{Zn}\left(\mathrm{mg} \mathrm{l}^{-1}\right)$ & $\begin{array}{c}0.82 \\
\pm 0.12\end{array}$ & $\begin{array}{c}1.28 \\
\pm 0.07\end{array}$ & $\begin{array}{c}0.72 \\
\pm 0.05\end{array}$ & $\begin{array}{c}1.17 \\
\pm 0.01\end{array}$ & $\begin{array}{c}0.82 \pm \\
0.12\end{array}$ & $\begin{array}{c}1.28 \\
\pm 0.07\end{array}$ & $\begin{array}{c}0.72 \\
\pm 0.05\end{array}$ & $\begin{array}{c}1.17 \\
\pm 0.01\end{array}$ \\
\hline
\end{tabular}

Each mean is across n number of replications $(n=3)$ 
Table 3. Summary of Analysis of Variance for water, soil and plant tissues for various attributes

\begin{tabular}{|c|c|c|c|c|c|c|c|c|c|c|c|c|c|c|c|c|}
\hline \multirow{4}{*}{$\begin{array}{c}\text { Water } \\
\text { Parameters }\end{array}$} & \multicolumn{16}{|c|}{ Water } \\
\hline & \multicolumn{4}{|c|}{ Site1 } & \multicolumn{4}{|c|}{ Site 2} & \multicolumn{4}{|c|}{ Site1 } & \multicolumn{4}{|c|}{ Site 2} \\
\hline & \multicolumn{4}{|c|}{ Sources } & \multicolumn{4}{|c|}{ Seasons } & \multicolumn{4}{|c|}{ Sources } & \multicolumn{4}{|c|}{ Seasons } \\
\hline & \multicolumn{2}{|c|}{ MS } & \multicolumn{2}{|c|}{$\mathrm{F}$} & \multicolumn{2}{|c|}{ MS } & \multicolumn{2}{|c|}{$\mathrm{F}$} & \multicolumn{2}{|c|}{ MS } & \multicolumn{2}{|l|}{$\mathrm{F}$} & \multicolumn{2}{|c|}{ MS } & \multicolumn{2}{|c|}{$\mathrm{F}$} \\
\hline $\mathrm{pH}$ & \multicolumn{2}{|c|}{3.876} & \multicolumn{2}{|c|}{$*$} & \multicolumn{2}{|c|}{0.140} & \multicolumn{2}{|c|}{ ns } & \multicolumn{2}{|c|}{3.172} & \multicolumn{2}{|c|}{$*$} & \multicolumn{2}{|c|}{0.969} & \multicolumn{2}{|c|}{$*$} \\
\hline $\mathrm{EC}$ & \multicolumn{2}{|c|}{0.001} & \multicolumn{2}{|c|}{$* * *$} & 0.1 & & $\mathrm{n}$ & & 0.0 & & $* *$ & & 0.0 & & $\mathrm{n}$ & \\
\hline $\mathrm{Na}^{+}$ & 0.3 & & $\mathrm{n}$ & & 24. & & $\mathrm{n}$ & & 4.9 & & $\mathrm{~ns}$ & & 224 & & $* *$ & \\
\hline $\mathrm{Cr}$ & 0.0 & & $\mathrm{n}$ & & 0.0 & & $\mathrm{n}$ & & 0.1 & & $* *$ & & 0.00 & & $\mathrm{n}$ & \\
\hline $\mathrm{Ni}$ & 0.1 & & $\mathrm{n}$ & & 3. & & $*:$ & & 0.0 & & * & & 0.00 & & $* *$ & \\
\hline $\mathrm{Pb}$ & 0.0 & & $* *$ & & 0.00 & & $\mathrm{n}$ & & 0.0 & & $* *$ & & 0.0 & & $\mathrm{n}$ & \\
\hline $\mathrm{Zn}$ & 0.6 & & $* *$ & & 0.0 & & $n$ & & 0.1 & & $* *$ & & 0.0 & & $\mathrm{n}$ & \\
\hline BOD & 5.1 & & $* *$ & & 82. & & $*:$ & & 2.2 & & $* *$ & & 16. & & $* *$ & \\
\hline COD & 4.2 & & $* *$ & & 3.6 & & $*$ & & 4.8 & & $* *$ & & 0.0 & & $\mathrm{n}$ & \\
\hline $\mathrm{Ca}^{+2}+\mathrm{Mg}^{+2}$ & 9.6 & & $\mathrm{n}$ & & 0.2 & & * & & 1.0 & & $* *$ & & 1.0 & & $* *$ & \\
\hline & & & & & & & & & oil & & & & & & & \\
\hline & & & & Depth & $10 \mathrm{~cm})$ & & & & & & & epth & $20 \mathrm{~cm})$ & & & \\
\hline $\begin{array}{c}\text { SoII } \\
\text { Parameters }\end{array}$ & & & & & & & & & & & & & & & & \\
\hline & Sea & & Spe & & Sea & & Spe & & Sea & & Spec & & Sea & & Spe & \\
\hline & MS & $\mathrm{F}$ & MS & $\mathrm{F}$ & MS & $\mathrm{F}$ & MS & $\mathrm{F}$ & MS & $\mathrm{F}$ & MS & $\mathrm{F}$ & MS & $\mathrm{F}$ & MS & $\mathrm{F}$ \\
\hline $\mathrm{pH}$ & 0.054 & $* * *$ & 0.010 & $* * *$ & 0.345 & $* * *$ & 0.107 & $* * *$ & 0.180 & $* * *$ & 0.072 & $* * *$ & 0.700 & $* * *$ & 0.353 & $* * *$ \\
\hline $\mathrm{EC}$ & 0.662 & ns & 11.642 & $* * *$ & 0.456 & $* * *$ & 2.707 & $* * *$ & 0.297 & $\mathrm{~ns}$ & 13.041 & $* * *$ & 0.307 & $* * *$ & 0.007 & $\mathrm{~ns}$ \\
\hline $\mathrm{Na}^{+}$ & 9.95 & ns & 7897.8 & $* * *$ & 94.3 & $* * *$ & 13263 & $* * *$ & 34.5 & $\mathrm{~ns}$ & 20758.4 & $* * *$ & 3.32 & $\mathrm{~ns}$ & 9366.6 & $* * *$ \\
\hline $\mathrm{Cr}$ & 0.014 & $* * *$ & 0.036 & $* * *$ & 0.007 & $* * *$ & 0.019 & $* * *$ & 0.016 & $* * *$ & 0.092 & $* * *$ & 0.010 & $* * *$ & 0.024 & $* * *$ \\
\hline $\mathrm{Ni}$ & 0.012 & $* * *$ & 0.006 & $*$ & 0.01 & ns & 0.05 & ns & 0.027 & $\mathrm{~ns}$ & 0.033 & $*$ & 0.187 & $* *$ & 0.0003 & $\mathrm{~ns}$ \\
\hline $\mathrm{Pb}$ & 0.019 & $* * *$ & 0.187 & $* * *$ & 0.014 & $* * *$ & 0.030 & $* * *$ & 0.004 & ns & 0,780 & $* * *$ & 0.010 & ns & 0.269 & $* * *$ \\
\hline $\mathrm{Zn}$ & 0.030 & $* *$ & 0.004 & $* * *$ & 0.010 & $* * *$ & 0.030 & $* *$ & 0.019 & $*$ & 0.187 & $* *$ & 0.014 & $*$ & 0.030 & $*$ \\
\hline
\end{tabular}




\begin{tabular}{|c|c|c|c|c|c|c|c|c|c|c|c|c|c|c|c|c|}
\hline \multirow{4}{*}{ Metals } & \multicolumn{16}{|c|}{ Plant tissues } \\
\hline & \multicolumn{4}{|c|}{ Roots } & \multicolumn{3}{|c|}{ Shoots } & & \multicolumn{4}{|c|}{ Leaves } & \multicolumn{4}{|c|}{ Pulp } \\
\hline & \multicolumn{2}{|c|}{ Sites } & \multicolumn{2}{|c|}{ Species } & \multicolumn{2}{|c|}{ Sites } & \multicolumn{2}{|c|}{ Species } & \multicolumn{2}{|c|}{ Sites } & \multicolumn{2}{|c|}{ Species } & \multicolumn{2}{|c|}{ Sites } & \multicolumn{2}{|c|}{ Species } \\
\hline & MS & $\mathrm{F}$ & MS & $\mathrm{F}$ & MS & $\mathrm{F}$ & MS & $\mathrm{F}$ & MS & $\mathrm{F}$ & MS & $\mathrm{F}$ & MS & $\mathrm{F}$ & MS & $\mathrm{F}$ \\
\hline $\mathrm{Cr}$ & 182.4 & $* * *$ & 632.94 & $* * *$ & 1237.87 & $*$ & 8220.44 & $* * *$ & 0.423 & $\mathrm{~ns}$ & 267.3 & $* * *$ & 1.36 & $\mathrm{~ns}$ & 2483.3 & $* *$ \\
\hline $\mathrm{Ni}$ & 266.5 & $*$ & 178.89 & $* *$ & 425.39 & $* *$ & 2654.83 & $*$ & 49.23 & $\mathrm{~ns}$ & 440.3 & $* *$ & 0.023 & ns & 520.60 & $*$ \\
\hline $\mathrm{Pb}$ & 382.2 & $*$ & 1509.32 & $*$ & 18.9 & $*$ & 13794.5 & $*$ & 182.25 & $* * *$ & 342.25 & $* *$ & 4.69 & ns & 2320.03 & $*$ \\
\hline $\mathrm{Zn}$ & 26.52 & $* *$ & 42.25 & $* * *$ & 13.14 & $*$ & 12.78 & $* *$ & 3.361 & ns & 14.69 & ns & 38.5 & ns & 61091.8 & $* * *$ \\
\hline
\end{tabular}

$\mathrm{MS}=$ mean square, $\mathrm{F}=\mathrm{F}$ value, $* * *, * *, *=$ Significant at $0.001,0.01$ and $0.05 \%$ level of probability, $\mathrm{ns}=$ non-significant by ANOVA, double factor with replication 
Table 4. Mean values ( \pm S. E) for physico - chemical and heavy metal contents of soil samples collected at depth $(0-10 \mathrm{~cm})$ from rhizospheres of two fruit species $(C$. reticulata and $M$. indica) irrigated with two different water sources during summer (May to October) and winter (November to March) at Site 1 (Aziz Farm) and Site 2 (Nasim Farm), Multan, Punjab, Pakistan

\begin{tabular}{|c|c|c|c|c|}
\hline \multirow{3}{*}{ Parameters } & \multicolumn{4}{|c|}{ Site 1 (Soil depth 0-10 cm) } \\
\hline & \multicolumn{2}{|c|}{ Summer } & \multicolumn{2}{|c|}{ Winter } \\
\hline & C. reticulata & M. indica & C. reticulata & M. indica \\
\hline $\mathrm{pH}$ & $8.26 \pm 0.01$ & $8.25 \pm 0.02$ & $8.17 \pm 0.01$ & $8.06 \pm 0.03$ \\
\hline $\mathrm{EC}\left(\mathrm{dS} \mathrm{m} \mathrm{m}^{-1}\right)$ & $3.51 \pm 0.06$ & $2.17 \pm 0,01$ & $4.61 \pm 1.02$ & $2.01 \pm 0.06$ \\
\hline $\mathrm{Na}^{+}\left(\mathrm{mg} \mathrm{l}^{-1}\right)$ & $182.89 \pm 13.30$ & $128.80 \pm 0.27$ & $181.93 \pm 0.35$ & $133.40 \pm 0.53$ \\
\hline $\mathrm{Cr}\left(\mathrm{mg} \mathrm{l}^{-1}\right)$ & $0.55 \pm 0.01$ & $0.66 \pm 0.00$ & $0.62 \pm 0.00$ & $0.73 \pm 0.00$ \\
\hline $\mathrm{Ni}\left(\mathrm{mg} \mathrm{l}^{-1}\right)$ & $0.82 \pm 0.01$ & $0.86 \pm 0.01$ & $0.88 \pm 0.01$ & $0.93 \pm 0.001$ \\
\hline $\mathrm{Pb}\left(\mathrm{mg} \mathrm{l}^{-1}\right)$ & $2.04 \pm 0.15$ & $1.28 \pm 0.05$ & $2.11 \pm 0.01$ & $1.35 \pm 0.00$ \\
\hline $\mathrm{Zn}\left(\mathrm{mg} \mathrm{l}^{-1}\right)$ & $0.61 \pm 0.01$ & $0.55 \pm 0.01$ & $0.69 \pm 0.00$ & $0.67 \pm 0.01$ \\
\hline \multicolumn{5}{|c|}{ Site 2 (Soil depth 0-10 cm) } \\
\hline $\mathrm{pH}$ & $8.40 \pm 0.03$ & $8.25 \pm 0.02$ & $8.10 \pm 0.06$ & $7.87 \pm 0.01$ \\
\hline $\mathrm{EC}\left(\mathrm{dS} \mathrm{m} \mathrm{m}^{-1}\right)$ & $2.60 \pm 0,03$ & $3.46 \pm 0.03$ & $2.12 \pm 0.06$ & $3.16 \pm 0.01$ \\
\hline $\mathrm{Na}^{+}\left(\mathrm{mg} \mathrm{l}^{-1}\right)$ & $130.11 \pm 0.90$ & $57.39 \pm 1.62$ & $129.49 \pm 0.27$ & $69.23 \pm 0.74$ \\
\hline $\mathrm{Cr}\left(\mathrm{mg} \mathrm{l}^{-1}\right)$ & $0.64 \pm 0.00$ & $0.71 \pm 0.01$ & $0.68 \pm 0.00$ & $0.77 \pm 0.01$ \\
\hline $\mathrm{Ni}\left(\mathrm{mg} \mathrm{l}^{-1}\right)$ & $1.12 \pm 0.12$ & $1.25 \pm 0.01$ & $1.18 \pm 0.00$ & $1.31 \pm 0.00$ \\
\hline $\mathrm{Pb}\left(\mathrm{mg} \mathrm{l}^{-1}\right)$ & $1.65 \pm 0.06$ & $1.41 \pm 0.02$ & $1.69 \pm 0.00$ & $1.42 \pm 0.00$ \\
\hline $\mathrm{Zn}\left(\mathrm{mg} \mathrm{l}^{-1}\right)$ & $0.63 \pm 0.00$ & $0.72 \pm 0.00$ & $0.68 \pm 0.00$ & $0.79 \pm 0.01$ \\
\hline
\end{tabular}

Each mean is across $n$ number of replications $(n=3)$

Table 5. Mean values ( \pm S. E) for physico - chemical and heavy metal contents of soil samples collected at depth $(10-20 \mathrm{~cm})$ from rhizospheres of two fruit species (C. reticulata and $M$. indica) irrigated with two different water sources during summer (May to October) and winter (November to March) at Site 1 (Aziz Farm) and Site 2 (Nasim Farm), Multan, Punjab, Pakistan

\begin{tabular}{|c|c|c|c|c|}
\hline \multirow{3}{*}{ Parameters } & \multicolumn{4}{|c|}{ Site 1 (Soil depth 0-10 cm) } \\
\hline & \multicolumn{2}{|c|}{ Summer } & \multicolumn{2}{|c|}{ Winter } \\
\hline & C. reticulata & M. indica & C. reticulata & M. indica \\
\hline $\mathrm{pH}$ & $8.60 \pm 0.03$ & $8.51 \pm 0.02$ & $8.42 \pm 0.00$ & $8.20 \pm 0.01$ \\
\hline $\mathrm{EC}\left(\mathrm{dS} \mathrm{m} \mathrm{m}^{-1}\right)$ & $3.58 \pm 0.06$ & $2.02 \pm 0.02$ & $4.42 \pm 0.99$ & $1.81 \pm 0.01$ \\
\hline $\mathrm{Na}^{+}\left(\mathrm{mg} \mathrm{l}^{-1}\right)$ & $202.01 \pm 18.87$ & $119.82 \pm 0.27$ & $206.54 \pm 0.96$ & $122.36 \pm 0.48$ \\
\hline $\mathrm{Cr}\left(\mathrm{mg} \mathrm{l}^{-1}\right)$ & $0.72 \pm 0.00$ & $0.91 \pm 0.02$ & $0.81 \pm 0.00$ & $0.97 \pm 0.01$ \\
\hline $\mathrm{Ni}\left(\mathrm{mg} \mathrm{l}^{-1}\right)$ & $1.01 \pm 0.06$ & $1.12 \pm 0.06$ & $1.11 \pm 0.00$ & $1.21 \pm 0.00$ \\
\hline $\mathrm{Pb}\left(\mathrm{mg} \mathrm{l}^{-1}\right)$ & $2.22 \pm 0.08$ & $1.82 \pm 0.04$ & $2.29 \pm 0.00$ & $1.67 \pm 0.00$ \\
\hline $\mathrm{Zn}\left(\mathrm{mg} \mathrm{l}^{-1}\right)$ & $0.78 \pm 0.01$ & $0.51 \pm 0.01$ & $0.84 \pm 0.00$ & $0.61 \pm 0.01$ \\
\hline \multicolumn{5}{|c|}{ Site 2 (Soil depth 0-10 cm) } \\
\hline $\mathrm{pH}$ & $8.60 \pm 0.03$ & $8.40 \pm 0.02$ & $8.20 \pm 0.03$ & $7.80 \pm 0.09$ \\
\hline $\mathrm{EC}\left(\mathrm{dS} \mathrm{m} \mathrm{m}^{-1}\right)$ & $2.35 \pm 0.01$ & $2.29 \pm 0.04$ & $2.02 \pm 0.03$ & $1.98 \pm 0.04$ \\
\hline $\mathrm{Na}^{+}\left(\mathrm{mg} \mathrm{l}^{-1}\right)$ & $118.99 \pm 9.00$ & $60.73 \pm 2.01$ & $117.66 \pm 0.62$ & $64.17 \pm 0.35$ \\
\hline $\mathrm{Cr}\left(\mathrm{mg} \mathrm{l}^{-1}\right)$ & $0.51 \pm 0.01$ & $0.42 \pm 0.00$ & $0.57 \pm 0.00$ & $0.48 \pm 0.00$ \\
\hline $\mathrm{Ni}\left(\mathrm{mg} \mathrm{l}^{-1}\right)$ & $1.13 \pm 0.11$ & $1.13 \pm 0.01$ & $1.39 \pm 0.00$ & $1.37 \pm 0.00$ \\
\hline $\mathrm{Pb}\left(\mathrm{mg} \mathrm{l}^{-1}\right)$ & $1.72 \pm 0.11$ & $1.42 \pm 0.03$ & $1.78 \pm 0.01$ & $1.48 \pm 0.00$ \\
\hline $\mathrm{Zn}\left(\mathrm{mg} \mathrm{l}^{-1}\right)$ & $0.64 \pm 0.00$ & $0.74 \pm 0.01$ & $0.71 \pm 0.01$ & $0.81 \pm 0.01$ \\
\hline
\end{tabular}

Each mean is across number of replications $(n=3)$ 
Table 2 revealed a temporal variation for EC in ground water of the two sites. Analysis of Variance (Table 3) depicted a highly significant $(\mathrm{P} \leq 0.001)$ variability for EC regarding irrigation sources but no temporal contrast at both sites. Electrical conductivity (EC) was greater in citrus root zone during winter at both soil depths of the two sites (Tables 4 and 5). Analysis of variance (Table 3) clearly indicated that soil rhizosphere of the species was highly significant $(\mathrm{P} \leq 0.001)$ for $\mathrm{EC}$ at both depths at site 1 but at site 2 , soil depths possessed a significant $(\mathrm{P} \leq 0.001)$ temporal disparity for EC.

A higher level of cations; sodium $\left(\mathrm{Na}^{+}\right)$and calcium, magnesium $\left(\mathrm{Ca}^{+2}+\mathrm{Mg}^{+2}\right)$ was recorded in tube well water during summer and winter, respectively at site 1 but the most elevated values for both cations were measured in canal water during summer at site 2 as presented in Table 2. Analysis of Variance (Table 3) depicted highly significant $(\mathrm{P} \leq 0.001)$ contrast for $\mathrm{Na}^{+}$regarding seasons at site 2 . Statistical analysis also showed marked differences $(\mathrm{P} \leq 0.01)$ for $\mathrm{Ca}^{+2}+\mathrm{Mg}^{+2}$ regarding seasons at site 1 but at site 2 , ANOVA revealed highly significant $(\mathrm{P} \leq 0.001)$ disparity for $\mathrm{Ca}^{+2}+\mathrm{Mg}^{+2}$ regarding sources and seasons. An elevated level of $\mathrm{Na}^{+}$was noticed at soil depth $(0-10 \mathrm{~cm})$ for citrus rhizosphere during summer at site 1 (Table 4) but more $\mathrm{Na}^{+}$was found at $10-20 \mathrm{~cm}$ of soil during winter (Table 5). The statistical analysis (Table 3) for $\mathrm{Na}^{+}$ depicted significant $(\mathrm{P} \leq 0.001)$ contrast for rhizosphere from two soil depths at both sites.

An elevated demand for biological and chemical oxygen was measured in ground water during winter at site 1 and 2, respectively (Table 2). Analysis of Variance (Table 3) for BOD and COD showed significant $(\mathrm{P} \leq 0.001)$ temporal variability as well as disparity between irrigation sources at site 1 but at site 2 , water sources were significantly $(\mathrm{P} \leq 0.001)$ variable for $\mathrm{BOD}$ and COD.

Heavy metals $(\mathrm{Cr}, \mathrm{Ni}, \mathrm{Pb}, \mathrm{Zn})$ in irrigation water showed (Table 2) higher concentration in ground water during summer except $\mathrm{Zn}$ with elevated levels during winter. Overall site 2 exhibited more metal contents. Statistical analysis (Table 3) revealed significant $(\mathrm{P} \leq 0.001)$ variability for $\mathrm{Cr}$ regarding water sources. Whereas, ANOVA for Ni depicted significant $(\mathrm{P} \leq 0.001)$ temporal variability at both sites. Analysis of Variance also depicted that the irrigation sources were highly significant $(\mathrm{P} \leq 0.001)$ for $\mathrm{Pb}$ and $\mathrm{Zn}$. A greater concentration of chromium $(\mathrm{Cr})$ was recorded in mango root zone at soil depth $0-10 \mathrm{~cm}$ during winter at site 1 (Table 4 ) but more $\mathrm{Cr}$ was detected in deeper soil layers (Table 5). Analysis of variance (Table 3) for Cr revealed highly significant $(\mathrm{P} \leq 0.001)$ spatial and temporal variability. A much higher level of nickel (Ni) was found at soil depth $0-10 \mathrm{~cm}$ for citrus rhizosphere during winter at site 1 (Table 4). At site 2, citrus rhizosphere at $10-20 \mathrm{~cm}$ soil depth had an elevated concentration of Ni during winter (Table 5). ANOVA (Table 3) revealed a significant $(\mathrm{P} \leq 0.05)$ contrast for Ni regarding rhizospheres of fruit species from both soil depths at site 1 . Whereas, at site $2(10-20 \mathrm{~cm}$ soil depth), ANOVA depicted significant $(\mathrm{P} \leq 0.001)$ temporal variability for $\mathrm{Ni}$. Greater concentrations of lead $(\mathrm{Pb})$ were recorded in citrus root zones at both soil layers at site 1 during winter (Tables 4 and 5). Statistical analysis (Table 3$)$ revealed significant $(\mathrm{P} \leq 0.001)$ spatially and temporally variable $\mathrm{Pb}$ content. Mean values for $\mathrm{Zn}$ presented in Table 4, depicted its higher level in citrus rhizosphere at $0-10 \mathrm{~cm}$ depth during winter at site 1 . On contrary, more $\mathrm{Zn}$ was found in mango root zone at 10-20 cm depth at site 2 (Table 5). Analysis of Variance (Table 3) revealed a significant $(\mathrm{P} \leq 0.001)$ disparity for $\mathrm{Zn}$ for species rhizosphere, seasons and soil depths. 


\section{Biochemical attributes of plant species}

Chlorophyll ' $a$ ' (Figure 3) exhibited the highest levels in citrus foliage at site 1 in contrast to mango leaves which had higher value for this pigment at site 2 . Figure 3 also depicted a distinct difference for chlorophyll ' $b$ ' in the leaves of two species at site 1 . It was also observed that chlorophyll ' $b$ ' was distinctively higher in citrus leaves. Yet at site 2 , no marked contrast was observed for chlorophyll ' $b$ '.
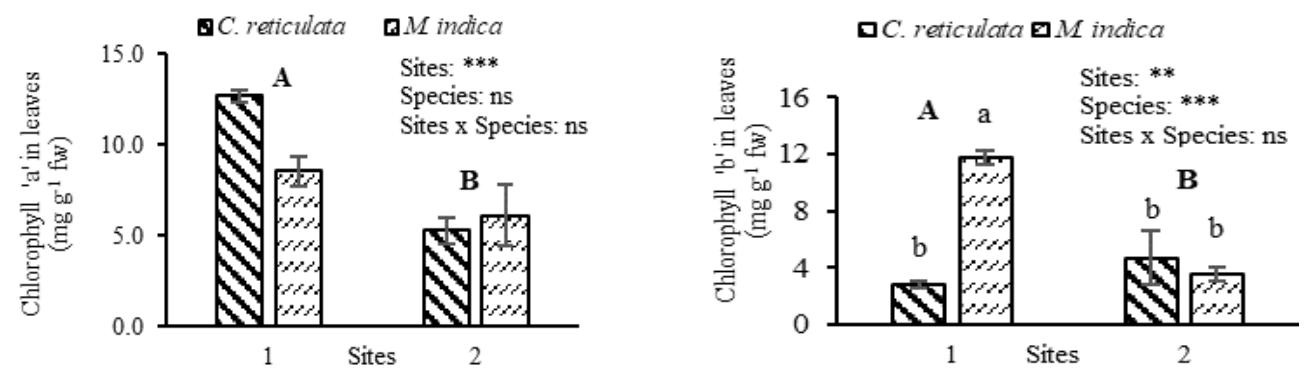

Figure 3. Mean values for Chlorophyll (' $a$ ' and ' $b$ ') in leaves of two fruit species (C. reticulata and $M$. indica) sampled during respective fruiting seasons at Site 1 (Aziz Farm) and Site 2 (Nasim Farm), Multan, Punjab, Pakistan. The comparison between sites with different letters A and $B$ and for species $a \& b$ showed a significant contrast. Each mean is across three individual trees with three foliage samples. Each column corresponds to mean value and each vertical line

represents \pm S.E. Different letters on each column showed significant difference by $L S D$

(Tukey's Test) at 5\% level of probability. ***,**,* = Significant at 0.001, 0.01 and $0.05 \%$ level of probability, $n s=$ non-significant by ANOVA, double factor with replication

\section{Extent of oxidative damage}

Mean values presented in Figure 4 revealed greater production of MDA in mango leaves. Although, MDA production was higher in mango foliage but it did not possess a distinct contrast from citrus. Similarly, the species were invariably different spatially. Analysis of variance (Figure 4) depicted that foliage had no noticeable difference between sites for MDA content. However, the foliage of the two species differed significantly $(\mathrm{P} \leq 0.05)$ for MDA content.

\section{Enzymatic antioxidative activity}

An enhanced activity for SOD was noticed that was considerably higher for citrus (Figure 4). A distinct variability for the activity SOD was observed in the two species from both sites. Though differential activity of CAT was noticed but the biosynthesis of the enzyme was insignificantly variable in the species (Figure 4). Hence, ANOVA (Figure 4) did not reveal any marked contrast for CAT activity for the foliage of the species. Conversely, at site 2, a profound contrast was observed for CAT level in the leaves of two species. With regard to POD, both species have indistinctive responses from both sites.

\section{Non enzymatic antioxidative activity}

A highly profound contrast was observed for ascorbic acid content in the leaves of two species but mango foliage had noticeably higher level for ascorbic acid. Despite variable mean values, no marked disparity became evident among the foliage of two 
species for ascorbic acid content at site 2 (Figure 5). Statistical analysis for ascorbic acid revealed that the sites and species varied $(\mathrm{P} \leq 0.001)$ considerably. Similarly, more carotenoids were found in mango leaves at site 1 (Figure 5) but a spatial contrast was evident. Furthermore, citrus foliage had lower amount of carotenoids as compared to mango leaves which did not exhibit any marked contrast between two species from the both sites. Analysis of Variance depicted significant $(\mathrm{P} \leq 0.01)$ spatial variability and the species also differed considerably $(\mathrm{P} \leq 0.01)$. A greater level of phenols (Figure 5) was recorded in mango foliage than citrus at both sites hence a spatial variability became evident. However, by contrast higher level of soluble sugar was recorded in citrus foliage than mango at both sites but the two species had a distinct contrast at site 1 (Figure 5). ANOVA for soluble sugars and phenols depicted significant $(\mathrm{P}<0.001)$ contrast among species.
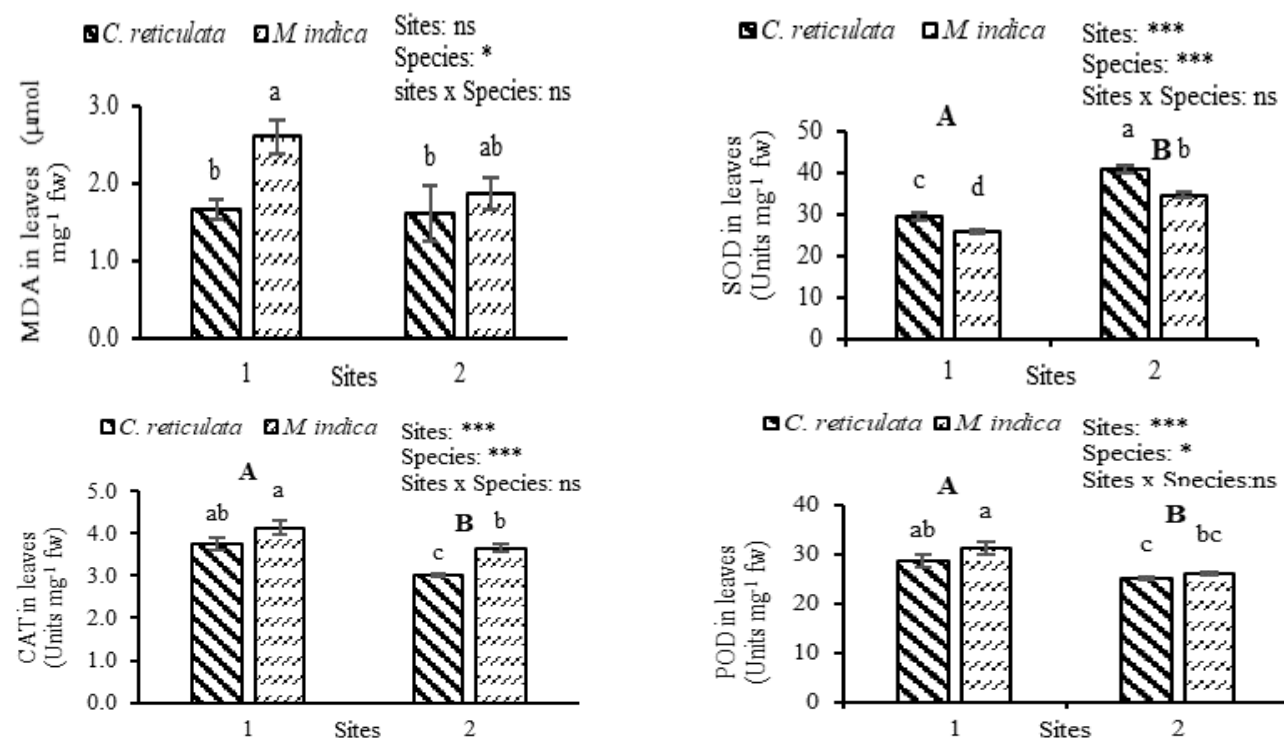

Figure 4. Mean values for MDA, SOD, CAT, POD in leaves of two fruit species (C. reticulata and $M$. indica) sampled during respective fruiting seasons at Site 1 (Aziz Farm) and Site 2

(Nasim Farm), Multan, Punjab, Pakistan. The comparison between sites with different letters A and $B$ and for species $a \& b$ or without common letter showed a significant contrast. Each mean is across three individual trees with three foliage samples. Each column corresponds to mean

value and each vertical line represents $\pm S$.E. Different letters on each column showed significant difference by LSD (Tukey's Test) at 5\% level of probability. ***, **, * = Significant at $0.001,0.01$ and $0.05 \%$ level of probability, $n s=$ non-significant by ANOVA, double factor with replication

\section{Heavy metals $(\mathrm{Cr}, \mathrm{Ni}, \mathrm{Pb}, \mathrm{Zn})$ transport and accumulation in plant tissues}

A substantial amount of metals was accumulated in roots and shoots of citrus plants as compared to mango which had more amount of metals in the foliage (Figure 6). Citrus fruit pulp has remarkably higher $\mathrm{Cr}, \mathrm{Ni}$ and $\mathrm{Pb}$ but traces of these metals were not found in the edible portions of mango. On contrary, $\mathrm{Zn}$ was found in the pulp of both fruit species but was much higher in citrus than that of mango. ANOVA (Table 3) depicted that $\mathrm{Cr}$ in roots, shoots and leaves was highly significant $(\mathrm{P} \leq 0.001)$ regarding 
species. $\mathrm{Pb}$ in leaves and $\mathrm{Zn}$ in pulp showed marked $(\mathrm{P} \leq 0.001)$ significance regarding sites and species, respectively.
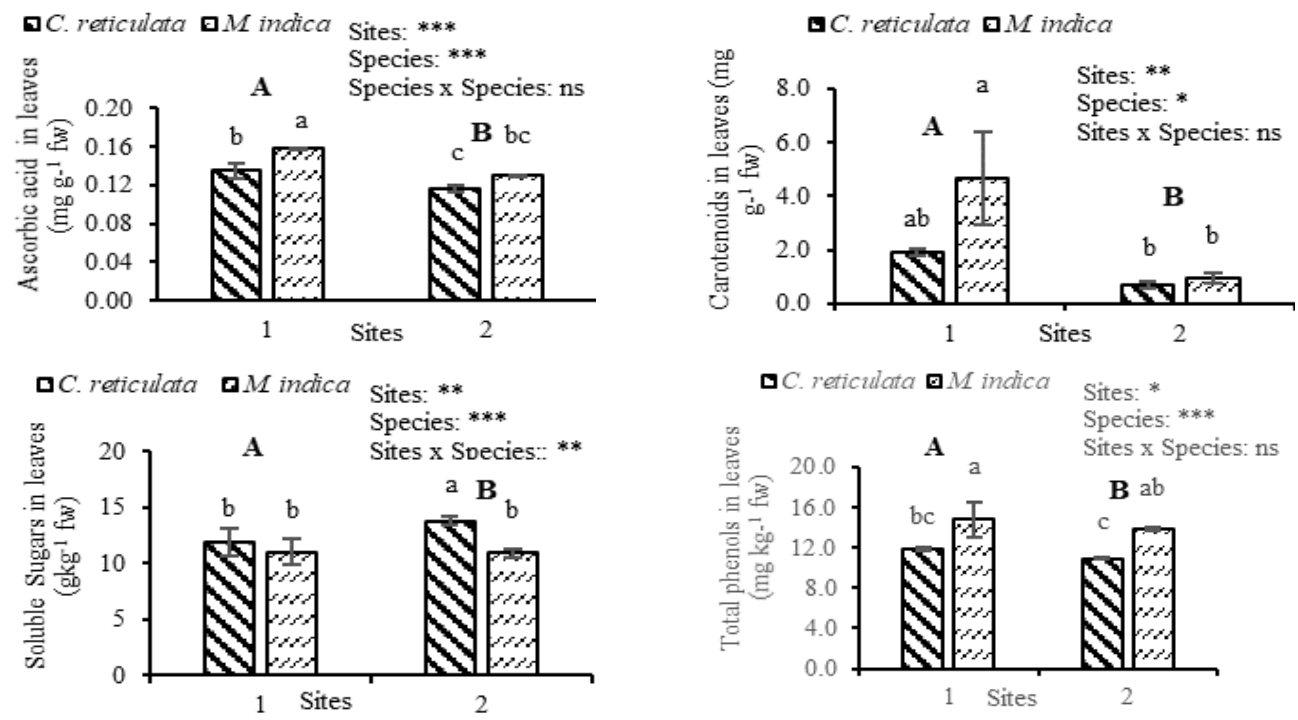

Figure 5. Mean values for non-enzymatic (ascorbic acid, carotenoids, soluble sugar, total phenolics) activities in leaves of two fruit species (C. reticulata and $M$. indica) sampled during respective fruiting seasons at Site 1 (Aziz Farm) and Site 2 (Nasim Farm), Multan, Punjab,

Pakistan. The comparison between sites with different letters $A$ and $B$ and for species $a \& b$ or without common letter showed a significant contrast. Each mean is across three individual trees with three foliage samples. Each column corresponds to mean value and each vertical line represents $\pm S$.E. Different letters on each column showed significant difference by $L S D$ (Tukey's Test) at 5\% level of probability. ***, **, * = Significant at $0.001,0.01$ and $0.05 \%$ level of probability, $n s=$ non-significant by ANOVA, double factor with replication
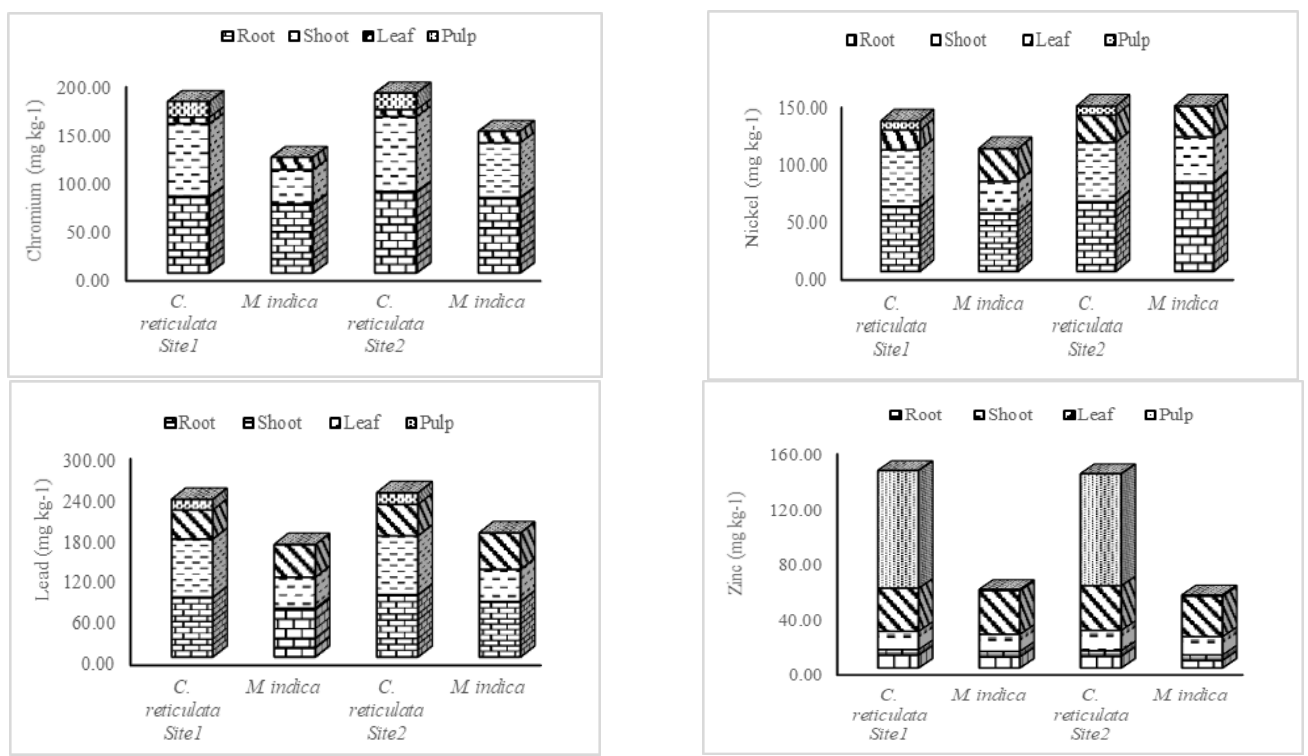

Figure 6. Mean values for heavy metals $(\mathrm{Cr}, \mathrm{Ni}, \mathrm{Pb}, \mathrm{Zn})$ in ( $\mathrm{C}$. reticulata and $\mathrm{M}$. indica) plant tissues (root, shoot, leaves, pulp) sampled from Site 1 (Aziz Farm) and Site 2 (Nasim Farm), Multan, Punjab, Pakistan. Each mean is across three individual trees with three samples for each plant tissue 


\section{Discussion}

The study primarily focused on the quality of irrigation water, changes in soil properties with particular reference to metal contamination and its impact on socioeconomic aspects and food safety. It is well documented that irrigation water whether rain or surface water plays an imperative role for recharging and quality of underground water (Zhang et al., 2016). The recharging ability and quality are important determinants for ground water to suggest its safe consumption (Hussain et al., 2017) because it influences soil properties.

The current study indicated that temporal variation affected the ground water quality and soil properties from the selected sites. Among various water quality parameters, $\mathrm{pH}$ is of prime consideration as far as solubility of minerals and metal uptake is concerned. $\mathrm{pH}$ of canal irrigation water was high during summer but the potential hydrogen value for ground water was more during winter. The reason for this variation can be attributed to various types of effluents that are thrown into canals by industries during peak working months of summer. However, less evaporation rate during winter months resulted in more deposition of substances in soil and ground water. The findings of our study are in lines to the results documented by Patel et al. (2015) who reported comparable results for high $\mathrm{pH}$ of waste water because of several dissolved substances. As far as soil $\mathrm{pH}$ is concerned, it influences several factors that alter the soil properties and plant growth as well as it governs nutrient availability and metal uptake by plants (Eid et al., 2018). The soil pH also showed spatial and temporal variability. According to Onwuka and Mang (2018) seasonal change of temperature affects the radiant energy of soil. This change in energy also caused significant disturbance in biochemical and physico-chemical attributes of soils, thus alter decomposition rate, release of minerals organic and inorganic components of soil.

Electrical Conductivity (EC), Biological Oxygen Demand (BOD) and Chemical Oxygen Demand (COD) were found to be higher in ground water during winter. Periodic un and inundation of canals and leaching of salts to ground water seemed to be a possible reason for changes in EC. When water pumps are installed at moderate depth of soil, a significant amount of dissolved salts are mixed with water that upon irrigation can change soil properties. Although soil texture is an important aspect in this regard but the two sites did not possess any marked disparity because sandy loam texture of the soil at two sites. Hussain et al. (2017) provided similar basis for changes in quality of surface and ground water. Application of irrigation water with high $\mathrm{pH}$ and $\mathrm{EC}$ for a long time significantly increase the EC of soil which might be due to increased exchangeable complexes in soil and certain reactions of alkaline hydrolysis. These findings are in line with the results of Romic et al. (2008) who attributed the same reasons. Further, higher BOD might result from characteristics and types of substances/pollutants that specifically allow multitude kinds of aerobic microflora thus more BOD.

The cations $\left(\mathrm{Na}^{+}\right.$and $\left.\mathrm{Ca}^{+2}+\mathrm{Mg}^{+2}\right)$ remained higher in canal water during summer. An elevated soil temperature in summer season resulted in more evaporation from soil surface leaving behind more salts thus more pH, EC and BOD (Tian et al., 2020). Soil $\mathrm{Na}^{+}$was higher during summer in citrus root zone but more was found in deeper soil layer with temporal variability. More levels of metals $(\mathrm{Cr}, \mathrm{Ni}$ and $\mathrm{Pb})$ in surface water during un-inundations of canals seems due to the absence of dilution factor.

With regard to heavy metals in ground water, more metals were found during summer with greater concentration of $\mathrm{Cr}$. The higher levels of $\mathrm{Cr}$ in ground water can 
be explained in terms greater solubility of the metal. However, bioavailability and phytotoxicity depends on various soil factors and $\mathrm{Cr}$ speciation $(\mathrm{Cr}$ III and $\mathrm{Cr} \mathrm{VI}$ ) but in the absence of any special transporters for both forms, the metal mostly retained by the plant roots and in soil. An increasing tendency was observed for $\mathrm{Cr}$ in deeper soil layers of mango rhizosphere. Other metals ( $\mathrm{Ni}, \mathrm{Pb}$ and $\mathrm{Zn}$ ) also followed an increasing trend in citrus root zone at soil depth $(10-20 \mathrm{~cm})$. Interestingly, all metals showed the increasing level from upper to deeper soil layer thus, showing the transfer of metals into deeper soil layers. The root morphology of the two species is also distinct that governs the extent of metals in the soil thus, different concentration of $\mathrm{Cr}, \mathrm{Pb}, \mathrm{Ni}$ and $\mathrm{Zn}$ in rhizosphere of the species.

The results of the study indicated an affirmative relationship between metal contents of the irrigation water and soil. The greater metal concentration in the deeper soil layer can pose serious threat owing to non-biodegradable nature and contact with plant roots. Dotaniya et al. (2017) also worked out for a relationship between quality of irrigation water and soil contamination. These workers also ascribed water contamination owing to presence of $\mathrm{Cr}$ in soil and vice versa.

Tripathi and Chaurasia (2020) demonstrated greater levels $\mathrm{Pb}$ in irrigation water from sites where extensive use of fertilizers and pesticides was administrated. The source of Ni pollution seems to be several vegetable oil/ghee industries throwing waste water or landfills (Lu et al., 2016). The use of metal pipes and gradual corrosion due to high $\mathrm{pH}$ of water may also result in higher Ni content of water and soil depending on the source of irrigation water. $\mathrm{Pb}$ concentration in soil was consistently higher and its persistence and transport in the deeper layers of soil became evident. Findings of the study are in contrary to the results documented by Cecchi et al. (2008) who reported decreasing level of $\mathrm{Pb}$ with increasing soil depth. However, behavior of $\mathrm{Pb}$ in the soil is governed by complex biogeochemical factors (Punamiya et al., 2010) including pH, cation exchange capacity, soil minerals, microbial flora, quantity of $\mathrm{Pb}$ in the soil and the type of plant species growing in the area. Nonetheless, polluted irrigation water mainly causes contamination of agricultural soils (Chaoua et al., 2019).

Zinc $(\mathrm{Zn})$ as an essential element which plays a pivotal role for various metabolic activities in plants (Hafeez et al., 2013) and is the most mobile element to be reached to aquifer. Sandy loam soils can allow percolation more $\mathrm{Zn}$ since soil act as sink for $\mathrm{Zn}$ hence an efficient uptake by plants becomes crucial. However, $\mathrm{Zn}$ uptake is not closely related to its concentration in the soil (Ernst and Nelissen, 2000) but the availability depends on soil $\mathrm{pH}$ and $\mathrm{P}$ status. The bioaccumulation of $\mathrm{Zn}$ in root cells and underlying mechanisms of its uptake are not completely known but its transport across the plasma membrane is mediated by a transport system and intracellular high-affinity binding sites (Gupta et al., 2016). Zn transporter family is comprised of ZIP (ZRT, IRT-like protein) are thought to be the primary transporters involved in its uptake. Thus, its accumulation in water and soil, subsequent transport and bioaccumulation in plants varied from other essential and non-essential metal ions (Guerinot, 2000). Nonetheless, the transport and bioaccumulation of $\mathrm{Zn}$ in edible part of the fruits remained of dietary significance. The results of the study are in accordance with the findings of Belkhiri et al. (2018).

The photosynthetic machinery comprising of green pigments chlorophyll ( $a$ and $b$ ) can serve as main predictors to signify net carbon assimilation via sunlight absorption by these molecules (Gruber et al., 2019). A species varied in space and time for chlorophyll $a$ and $b$. However, the lowest content of chlorophyll $(a)$ was found in mango leaves while chlorophyll $(b)$ was highly masked in citrus leaves. The amount of 
chlorophyll decreases in relation to environmental disturbances where metal contaminants have widely been reported (Houri et al., 2020) to cause biodegradation of these molecules or replacement of metal ions with central magnesium thereby changing the chemistry of photosynthetic pigments.

MDA is a broadly used as a marker and index of oxidative damage that is destruction of biological membranes (Shahid et al., 2017). Therefore, Lou et al. (2017) have investigated MDA to assess physiological damage under various stressful conditions. The analysis of assays from the leaves of citrus and mango revealed more MDA content but the foliage of former species with greater extent of lipid peroxidation. It is highly likely that this oxidative damage is due to the presence of exceeding amount of heavy metals in the growth medium. Nonetheless, differential survival ability of the species allows them to mitigate metal stress through altered biochemical pathways for cellular defense. This adaptability of the living system that controls and regulates the generation of several enzymatic components (SOD, CAT, POD) and some non-enzymatic metabolites (ascorbic acid, carotenoids, phenolics and soluble sugars) are important (Shah et al., 2020). An increased activity of SOD in citrus leaves but more production of CAT and POD in mango foliage indicated oxidative defense in both species However, enzymatic components can work more efficiently if their biosynthesis occur in a cocurrent manner. Biosynthesis of CAT and POD in citrus seemed to be insufficient to mitigate oxidative damage. However, a sequential production of CAT followed by POD can scavenge free radicals thereby protecting cellular membranes in the mango leaves.

Ascorbic acid (AsA) is a vital, non-enzymatic antioxidant having considerable ability to scavenge ROS (Bilska et al., 2019). The presence of elevated levels of ascorbic acid in mango foliage seemed to mitigate ROS thus, protecting the species against heavy metals present in environment. Similarly, a group of pigments called carotenoids are important components and are capable to destroy ROS, eliminate the excited triplet state of chlorophyll hence play a defensive role for chloroplasts integrity by inhibiting cracking of its membranes (Handa et al., 2018). M. indica innately possess several secondary metabolites in different plant parts including carotenoids thus seems to have a better defense where an affirmative relationship was observed between resilience and biosynthesis of carotenoids (Ahmad et al., 2010).

Phenolic compounds also have the same ability. These components make the species less deterrent to insects and pest attack. Again, $M$. indica is characterized by the presence of many phenolic compounds that are species specific and are genetically controlled. Nevertheless, a confirmatory relationship between environmental constraints and phenolics seems to provide a better resilience to the species. Thus, high contents of phenols in the mango family are considered as selective advantage for superior survival ability (Sharma et al., 2019) for various abiotic and biotic stresses.

Likewise, sugars regulate many physiological processes such as respiration, seed germination, flowering, senescence, and act as the most efficient osmo-regulator under various environmental stresses (Sami et al., 2016). In the present study, sugar metabolism did not differ considerably among fruit species. Therefore, non- enzymatic antioxidants other than soluble sugars seemed to have greater impact against heavy metal stress.

Since, all plants have the natural ability to extract metal from the roots to their aerial parts that successively decreases from below ground to above ground but the least contents are found in the fruits and seeds. Nevertheless, these limits are recommended by WHO (2012) to ensure food safety. 
A differential pattern of metal bioaccumulation in plant parts of the two species from two sites became evident. The species and sites exhibited a striking contrast $(\mathrm{p} \leq 0.001)$ for the bioaccumulation of different metals in plant parts except for the amount of metal in pulp where sites had shown no variability for all four metals. Similarly, Zn content in the foliage in both species and sites had no marked variation (Table 3). All plant tissues; roots, shoots and leaves, $(\mathrm{p} \leq 0.001)$ and edible part/ pulp $(\mathrm{p} \leq 0.01)$ in the two species varied considerably. However, the two sites differed significantly for metal contents except for pulp. The bioaccumulation and subsequent translocation of $\mathrm{Cr}$ was root > shoot < leaves < fruit pulp that is a gradual decline for translocation to above ground plant parts. The behavior of $\mathrm{Ni}$ and $\mathrm{Pb}$ uptake was comparable to that of $\mathrm{Cr}$ despite structural differences of these ions and differential transport mechanism (Chen et al., 2009). On the other hand, $\mathrm{Zn}$ transport and accumulation were strikingly different.

In plants, Zinc is of immense importance in fruit setting, quality and yield (Nasir et al., 2016). Zinc transport from root to fruit pulp is of dietary significance particularly in the absence of any visible phototoxic symptoms in fruit species that are characteristically associated with supra optimal levels of $\mathrm{Zn}$. A number of health disorder are associated with Zn deficiency but fruit pulps exhibited ample amount of this essential element (Rahimi et al., 2017).

The detection of $\mathrm{Cr}$ in citrus pulp signifies its bioaccumulation in edible portion is parallel to many studies (Omoyajowo et al., 2017). On the other hand, transport and bioaccumulation of $\mathrm{Cr}$ in mango appeared to be restricted hence the pulp remained metal free which is in line with Ogunkunle et al. (2014) who reported complete absence of $\mathrm{Cr}$ in various fruit species. Likewise, higher levels of $\mathrm{Pb}$ citrus pulp found consistent with other researchers (Saleh et al., 2017) who well reported the presence of $\mathrm{Pb}$ in a number of fruits across the globe. However, no $\mathrm{Pb}$ was found in mango pulp which is advantageous. A number of workers (Pereira et al., 2014; Sajib et al., 2014; Vollmannova et al., 2015) also reported the absence of $\mathrm{Pb}$ in many fruits such as Psidium guajava (guava), Litchi chinensis (litchi), Butia capitata (pindo palm) and Rubus idaeus (raspberry) owing to the natural ability or specific mechanism for limited transport for least bioaccumulation of metals in edible portion.

The differential occurrence of heavy metal contents in the pulp of two species can be explained in the light of distinct phenology of the two species. With regard to mango, the development of fruit encompasses only a few months as lax panicle (characteristic inflorescence) appeared during March and fruits became ready to harvest from May onwards depending on the variety. However, picking and packaging is completed by August. Thus, the exposure time of the fruit to the growth medium is limited. On the other hand, the floral development in citrus is initiated soon after the picking of fruit during October, each year. The fruits development is initiated within a few weeks after flowering. However, the fruits remained on the plants till maturity in the next season. This long-term exposure of the fruit to medium may result in the uptake and transport of more metals in edible parts of the fruit.

\section{Conclusion}

The results of the study signified spatial and temporal variability in the quality of irrigation water and soil characteristics. Though, the two sites did not possess much contrast presumably due to the absence of geographical isolation and similar interacting 
environmental variables. Nevertheless, soil and ground water quality appeared to be altered by the quality of surface water.

The photosynthetic biomolecules varied in species but the trait appeared to be genetically controlled. The ROS production and subsequent enzymatic and nonenzymatic oxidative defense seems to exist in both species but carotenoids and phenols provided more selective advantage to mango to scavenge metal stress.

The study depicted bioaccumulation of heavy metals in edible portion of the citrus only without any potential threat to mango as far as food safety is concerned. Differential metal contaminants can definitely be attributed to phenological traits of the two species. The study suggested strict environmental monitoring for the biodiversity and conservation in the region. At present, quality of water and soil did not seem to be of potential risk but if mounting pollution continues, it can affect sustainability with drastic ecological and socio-economic impacts. The current study signified that functional proteomics and genomics should be addressed for future studies to reveal insights of underlying molecular mechanism/s.

Acknowledgements. The work presented is the part of Ph.D thesis of the first author. The help rendered by Mr. Muhammad Khalid Rasheed, Scientific Officer, Soil and Water Testing Laboratory, Agriculture Department, Multan is greatly acknowledged for physioco-chemical analysis of soil and water samples.

\section{REFERENCES}

[1] Ahmad, P., Jaleel, C. A., Salem, M. A., Nabi, G., Sharma, S. (2010): Roles of enzymatic and nonenzymatic antioxidants in plants during abiotic stress. - Critical Reviews in Biotechnology 30(3): 161-175.

[2] Ainsworth, E. A., Gillespie, K. M. (2007): Estimation of total phenolic content and other oxidation substrates in plant tissues using Folin-Ciocalteu reagent. - Nature Protocols 2(4): 875-877.

[3] Amjad, M., Raza, H., Murtaza, B., Abbas, G., Imran, M., Shahid, M., Naeem, M. A., Zakir, A., Iqbal, M. M. (2020): Nickel Toxicity Induced Changes in Nutrient Dynamics and Antioxidant Profiling in Two Maize (Zea mays L.) Hybrids. - Plants 9(5): 1-15.

[4] Arnon, D. I. (1949): Copper enzymes in isolated chloroplasts: Polyphenol-oxidases in Beta vulgaris. - Plant Physiology 24(1): 1-15.

[5] Arora, M., Kiran, B., Rani, S., Rani, A., Kaur, B., Mittal, N. (2008): Heavy metal accumulation in vegetables irrigated with water from different sources. - Food chemistry 111(4): 811-815.

[6] Belkhiri, L., Tiri, A., Mouni, L. (2018): Assessment of Heavy Metals Contamination in Groundwater: A Case Study of the South of Setif Area, East Algeria. - In: Achievements and Challenges of Integrated River Basin Management, pp.17-31.

[7] Bilska, K., Wojciechowska, N., Alipour, S., Kalemba, E. M. (2019): Ascorbic acid-The little-known antioxidant in woody plants. - Antioxidants 8(12): 645.

[8] Boquete, M. T., Lang, I., Weidinger, M., Richards, C. L., Alonso, C. (2021): Patterns and mechanisms of heavy metal accumulation and tolerance in two terrestrial moss species with contrasting habitat specialization. - Environmental and Experimental Botany 182: 104336.

[9] Cakmak, I., Horst, W. J. (1991): Effect of aluminium on lipid peroxidation, superoxide dismutase, catalase and peroxidase activities in root tips of soyabean (Glycine max). Journal of Plant Physiology 83: 463-468. 
[10] Cecchi, M., Dumat, C., Alric, A., Felix-Faure, B., Pradere, P., Guiresse, M. (2008): Multi-metal contamination of a calcic cambisol by fallout from a lead-recycling plant. Geoderma 144(1-2): 287-298.

[11] Chance, B., Maehly, A. C. (1995): An assay of catalase and peroxidase. - In: Colowick, S. P., Kapland, N. D. (eds.) Method in Enzymology, Academic Press, New York.

[12] Chaoua, S., Boussaa, S., El Gharmali, A., Boumezzough, A. (2019): Impact of irrigation with wastewater on accumulation of heavy metals in soil and crops in the region of Marrakech in Morocco. - Journal of the Saudi Society of Agricultural Sciences 18(4): 429-436.

[13] Chaudhry, Q. U. (2017): Climate change profile of Pakistan. - Asian Development Bank, $116 \mathrm{p}$.

[14] Chen, M., Ma, L. Q. (2001): Comparison of three Aqua Regia digestion methods for twenty Florida soils. - Soil Science Society of America Journal 65: 491-499.

[15] Chen, C., Huang, D., Liu, J. (2009): Functions and toxicity of nickel in plants: recent advances and future prospects. - Clean-Soil, Air, Water 37(4-5): 304-313.

[16] Dotaniya, M., Meena, V., Rajendiran, S., Coumar, M. V., Saha, J., Kundu, S., Patra, A. (2017): Geo-accumulation indices of heavy metals in soil and groundwater of Kanpur, India under long term irrigation of tannery effluent. - Bulletin of Environmental Contamination and Toxicology 98(5): 706-711.

[17] Eid, E. M., Alrumman, S. A., Galal, T. M., El-Bebany, A. F. (2018): Prediction models for evaluating the heavy metal uptake by spinach (Spinacia oleracea L.) from soil amended with sewage sludge. - International Journal of Phytoremediation 20(14): 14181426.

[18] Ernst, W. H. O., Nelissen, H. J. M. (2000): Life-cycle phases of a zinc-and cadmiumresistant ecotype of Silene vulgaris in risk assessment of polymetallic mine soils. Environmental Pollution 107(3): 329-338.

[19] Estefan, G., Sommer, R., Ryan, J. (2013): Methods of soil, plant, and water analysis. - A Manual for the West Asia and North Africa Region 3: 65-119.

[20] Giannopolitis, C. N., Ries, S. K. (1977): Superoxide dismutase I. Occurrence in higher plants. - Plant Physiology 59: 309-314.

[21] GOP (Government of Pakistan). (2016): Pakistan statistical year book. - Statistics Division, Pakistan Bureau of Statistics, Government of Pakistan. http://www.pbs.gov.pk/content/pakistan-statistical-year-book-2016.

[22] Gruber, E., Kjær, C., Nielsen, S. B., Andersen, L. H. (2019): Intrinsic Photophysics of Light-harvesting Charge-tagged Chlorophyll a and b Pigments. - Chemistry-A European Journal 25(39): 9153-9158.

[23] Guerinot, M. L. (2000): The ZIP family of metal transporters. - Biochimica et Biophysica Acta (BBA) - Biomembranes 1465(1-2): 190-198.

[24] Gupta, N., Ram, H., Kumar, B. (2016): Mechanism of Zinc absorption in plants: uptake, transport, translocation and accumulation. - Reviews in Environmental Science and Bio/Technology 15(1): 89-109.

[25] Gupta, G. S., Yadav, G., Tiwari, S. (2020): Bioremediation of heavy metals: a new approach to sustainable agriculture. - In: Restoration of wetland ecosystem: a trajectory towards a sustainable environment. Springer, Singapore, pp.195-226.

[26] Hafeez, B. M., Khanif, Y., Saleem, M. (2013): Role of Zinc in Plant Nutrition- A Review. - Journal of Experimental Agriculture International 3(2): 374-391.

[27] Handa, N., Kohli, S., Sharma, A., Thukral, A., Bhardwaj, R., Alyemeni, M., Wijaya, L., Ahmad, P. (2018): Selenium ameliorates chromium toxicity through modifications in pigment system, antioxidative capacity, osmotic system, and metal chelators in Brassica juncea seedlings. - South African Journal of Botany 119: 1-10.

[28] Houri, T., Khairallah, Y., Al Zahab, A., Osta, B., Romanos, D., Haddad, G. (2020): Heavy metals accumulation effects on the photosynthetic performance of geophytes in Mediterranean reserve. - Journal of King Saud University-Science 32(1): 874-880. 
[29] Hu, B. F., Chen, S. C., Hu, J., Xia, F., Xu, J. F., Li, Y., Shi, Z. (2017): Application of portable XRF and VNIR sensors for rapid assessment of soil heavy metal pollution. PLoS ONE 12(2): e0172438.

[30] Hussain, Y., Ullah, S. F., Hussain, M. B., Aslam, A. Q., Akhter, G., Martinez-Carvajal, H., Cardenas-Soto, M. (2017): Modelling the vulnerability of groundwater to contamination in an unconfined alluvial aquifer in Pakistan. - Environmental Earth Sciences 76(2): 1-11.

[31] Ibrahim, M. A., Khan, P. R., Hegazy, S. S., Hashim, E. A., Azamal, H., Ansari, M. K. A., Altaf, A., Muhammad, I., Hakeem, K. R. (2015): Improving the phytoextraction capacity of plants to scavenge heavy-metalinfested sites. - Environmental Reviews 23: 1-22.

[32] Jirka, A. M., Carter, M. J. (1975): Micro semiautomated analysis of surface and waste waters for chemical oxygen demand. - Analytical Chemistry 47(8): 1397-1402.

[33] Keller, T., Schwager, H. (1977): Air pollution and ascorbic acid. - European Journal of Forest Pathology 7(6): 338-350.

[34] Leoveanu-Soare, B. E., Petre, L. I., Micu, M. M. (2020): Social and economic aspects regarding the development of agriculture in Romania. - Scientific Papers SeriesManagement, Economic Engineering in Agriculture and Rural Development 20(2): 281287.

[35] Lichtenthaler, H. K. (1987): Chlorophylls and carotenoids: pigments of photosynthetic biomembranes. - Methods in Enzymology 148: 350-382.

[36] Lou, Y., Zhao, P., Wang, D., Amombo, E., Sun, X., Wang, H., Zhuge, Y. (2017): Germination, physiological responses and gene expression of tall fescue (Festuca arundinacea Schreb.) growing under $\mathrm{Pb}$ and $\mathrm{Cd}$. - PloS One 12(1): e0169495.

[37] Lu, Y., Tang, C., Chen, J., Yao, H. (2016): Assessment of major ions and heavy metals in groundwater: a case study from Guangzhou and Zhuhai of the Pearl River Delta, China. Frontiers of Earth Science 10(2): 340-351.

[38] Mahfooz, Y., Yasar, A., Guijian, L., Islam, Q. U., Akhtar, A. B. T., Rasheed, R., Irshad, S., Naeem, U. (2020): Critical risk analysis of metals toxicity in wastewater irrigated soil and crops: a study of a semi-arid developing region. - Scientific Reports 10(1): 1-10.

[39] Medveckiene, B., Kulaitienè, J., Jarienè, E., Vaitkevičienė, N., Hallman, E. (2020): Carotenoids, Polyphenols, and Ascorbic Acid in Organic Rosehips (Rosa spp.) Cultivated in Lithuania. - Applied Sciences 10(15): 5337.

[40] Moore, P. D., Chapman, S. B. (1986): Methods in plant ecology. $-2^{\text {nd }}$ ed. Blackwell Scientific Publications.

[41] Muniraja, M., Vijayalakshmi, G., Naik, M. L., Terry, R., Khan, P. S. V. (2019): Ultrastructural observations of anthers, staminoids and pollen grains of Mango (Mangifera indica L. var. Beneshan; Anacardiaceae). - Palynology 44(4): 565-574.

[42] Nai, C., Tang, M., Liu, Y., Xu, Y., Dong, L., Liu, J., Huang, Q. (2021): Potentially contamination and health risk to shallow groundwater caused by closed industrial solid waste landfills: Site reclamation evaluation strategies. - Journal of Cleaner Production 286: 125402.

[43] Nasir, M., Khan, A. S., Basra, S. A., Malik, A. U. (2016): Foliar application of moringa leaf extract, potassium and zinc influence yield and fruit quality of 'Kinnow'mandarin. Scientia horticulturae 210: 227-235.

[44] Naz, S., Shahzadi, K., Rashid, S., Saleem, F., Zafarullah, A., Ahmad, S. (2014): Molecular characterization and phylogenetic relationship of different citrus varieties of Pakistan. - Journal of Animal and Plant Sciences 24(1): 315-320.

[45] NoorMmemon, M. I., Noonari, S., Sidhu, M. Y., Arain, M. U., Jamali, R. H., Mirani, A. A., Khajjak, A. K., Sial, S. A., Jamali, R., Jamro, A. H. (2015): Economic Analysis of Mango Orchard Production under Contrack Farming in Taluka Tando Adam District Sanghar Sindh, Pakistan. - Economics 5(11): 24-36. 
[46] Ogunkunle, A. T. J., Bello, O. S., Ojofeitimi, O. S. (2014): Determination of heavy metal contamination of street-vended fruits and vegetables in Lagos state, Nigeria. International Food Research Journal 21(5): 2115-2120.

[47] Omoyajowo, K. O., Njoku, K. L., Babalola, O. O., Adenekan, O. A. (2017): Nutritional composition and heavy metal content of selected fruits in Nigeria. - Journal of Agriculture and Environment for International Development 111(1): 123-139.

[48] Onwuka, B., Mang, B. (2018): Effects of soil temperature on some soil properties and plant growth. - Advances in Plants and Agriculture Research 8: 34-37.

[49] Patel, R., Tajddin, K., Patel, A., Patel, B. (2015): Physico-chemical analysis of textile effluent. - International Journal of Research and Scientific Innovation 5(2): 33-37.

[50] Pereira, M. C., Boschetti, W., Rampazzo, R., Celso, P. G., Hertz, P. F., Rios, A. D. O., Vizzotto, M., Flores, S. H. (2014): Mineral characterization of native fruits from the southern region of Brazil. - Food Science and Technology 34(2): 258-266.

[51] Prud'homme, M. P., Gonzalez, B., Billard, J. P., Boucaud, J. (1992): Carbohydrate content, fructan and sucrose enzyme activities in roots, stubble and leaves of ryegrass (Lolium perenne L.) as affected by source/sink modification after cutting. - Journal of Plant Physiology 140(3): 282-291.

[52] Punamiya, P., Datta, R., Sarkar, D., Barber, S., Patel, M., Das, P. (2010): Symbiotic role of glomus mosseae in phytoextraction of lead in vetiver grass [Chrysopogon zizanioides (L.)]. - Journal of Hazardous Materials 177(1-3): 465-474.

[53] Rahimi, G., Kolahchi, Z., Charkhabi, A. (2017): Uptake and translocation of some heavy metals by rice crop (Oryza sativa) in paddy soils. - Agriculture 63(4): 163-175.

[54] Randhawa, M. A., Ahmad, G., Anjum, F. M., Asghar, A., Sajid, M. W. (2014): Heavy metal contents and their daily intake in vegetables under peri-urban farming system of Multan, Pakistan. - Pakistan Journal of Agricultural Sciences 51(4): 1025-1031.

[55] Rehman, A., Alam, M., Malik, A., Ali, H., Sarfraz, B. (2015): Preharvest factors influencing the postharvest disease development and fruit quality of mango. - Journal of Environmental and Agricultural Sciences 3: 42-47.

[56] Richards, L. A. (1954): Diagnosis and Improvement of Saline and Alkali Soils, Agriculture. - Handbook 60, US Department of Agriculture, Riverside, California, USA.

[57] Romic, D., Ondrasek, G., Romic, M., Josip, B., Vranjes, M., Petosic, D. (2008): Salinity and irrigation method affect crop yield and soil quality in watermelon (Citrullus lanatus L.) growing. - Irrigation and Drainage: - The Journal of the International Commission on Irrigation and Drainage 57(4): 463-469.

[58] Sabir, M., Waraich, E. A., Hakeem, K. R., Ozturk, M., Ahmad, H. R., Shahid, M. (2015): Phytoremediation: mechanisms and adaptations. - In: Hakeem, K., Sabir, M., Ozturk, M., Mermut, A. (eds.) Soil remediation and plants: prospects and challenges. - Elsevier, New York, pp.85-105.

[59] Sajib, M. A. M., Jahan, S., Islam, M. Z., Khan, T. A., Saha, B. K. (2014): Nutritional evaluation and heavy metals content of selected tropical fruits in Bangladesh. International Food Research Journal 21(2): 609-615.

[60] Saleh, R., Cheraghi, M., Lorestani, B. (2017): Health assessment of heavy metal pollution (cadmium, lead, arsenic) in citrus marketed in Tehran, Iran, 2015. - Archives of Hygiene Sciences 6(2): 171-177.

[61] Sami, F., Yusuf, M., Faizan, M., Faraz, A., Hayat, S. (2016): Role of sugars under abiotic stress. - Plant Physiology and Biochemistry 109: 54-61.

[62] Shah, A. A., Ahmed, S., Ali, A., Yasin, N. A. (2020): 2-Hydroxymelatonin mitigates cadmium stress in cucumis sativus seedlings: Modulation of antioxidant enzymes and polyamines. - Chemosphere 243: 1-9.

[63] Shahid, M., Shamshad, S., Rafiq, M., Khalid, S., Bibi, I., Niazi, N. K., Dumat, C., Rashid, M. I. (2017): Chromium speciation, bioavailability, uptake, toxicity and detoxification in soil-plant system: A review. - Chemosphere 178: 513-533. 
[64] Sharma, A., Shahzad, B., Rehman, A., Bhardwaj, R., Landi, M., Zheng, B. (2019): Response of phenylpropanoid pathway and the role of polyphenols in plants under abiotic stress. - Molecules 24(13): 2452.

[65] Tian, F., Hou, M., Qiu, Y., Zhang, T., Yuan, Y. (2020): Salinity stress effects on transpiration and plant growth under different salinity soil levels based on thermal infrared remote (TIR) technique. - Geoderma 357: 1-10.

[66] Tirani, M. M., Haghjou, M. M. (2019): Reactive oxygen species (ROS), total antioxidant capacity (AOC) and malondialdehyde (MDA) make a triangle in evaluation of zinc stress extension. - Journal of Animal and Plant Sciences 29: 1100-1111.

[67] Tripathi, S. M., Chaurasia, S. (2020): Detection of Chromium in surface and groundwater and its bio-absorption using bio-wastes and vermiculite. - Engineering Science and Technology 23(5): 1153-1161.

[68] Tukey, J. W. (1949): Comparing individual means in the analysis of variance. Biometrics 5(2): 99-114.

[69] Vollmannova, A., Zupka, S., Bajcan, D., Medvecky, M., Daniel, J. (2015): Dangerous heavy metals in soil and small forest fruit as a result of old environmental loads. - In: Proceedings of the $14^{\text {th }}$ International Conference on Environmental Science and Technology, Rhodes, Greece, p.528.

[70] Wei, F. S. (2002): The standard methods for examination and analysis of water and wastewater, $4^{\text {th }}$ edition. - Beijing China Environmental Science Press.

[71] World Health Organization (WHO). (2012): Global Health Observatory (GHO) 2012. World Health Organization, Geneva.

[72] Zhang, B., Song, X., Zhang, Y., Ma, Y., Tang, C., Yang, L., Wang, Z. L. (2016): The interaction between surface water and groundwater and its effect on water quality in the Second Songhua River basin, northeast China. - Journal of Earth System Science 125(7): 1495-1507. 